\title{
POLAR DECOMPOSITION UNDER PERTURBATIONS OF THE SCALAR PRODUCT*
}

\author{
GUSTAVO $\mathrm{CORACH}^{\dagger}$, ALEJANDRA MAESTRIPIERI ${ }^{\ddagger}$, AND DEMETRIO STOJANOFF $\S$ \\ Dedicated to our friend and teacher Angel Rafael Larotonda \\ on his sixtieth birthday
}

\begin{abstract}
Let $\mathcal{A}$ be a unital $\mathrm{C}^{*}$-algebra with involution $*$ represented in a Hilbert space $\mathcal{H}, G$ the group of invertible elements of $\mathcal{A}, \mathcal{U}$ the unitary group of $\mathcal{A}, G^{s}$ the set of invertible selfadjoint elements of $\mathcal{A}, Q=\left\{\varepsilon \in G: \varepsilon^{2}=1\right\}$ the space of reflections and $P=Q \cap \mathcal{U}$. For any positive $a \in G$ consider the $a$-unitary group $\mathcal{U}_{a}=\left\{g \in G: a^{-1} g^{*} a=g^{-1}\right\}$, i.e., the elements which are unitary with respect to the scalar product $\langle\xi, \eta\rangle_{a}=\langle a \xi, \eta\rangle$ for $\xi, \eta \in \mathcal{H}$. If $\pi$ denotes the map that assigns to each invertible element its unitary part in the polar decomposition, it is shown that the restriction $\left.\pi\right|_{\mathcal{U}_{a}}: \mathcal{U}_{a} \rightarrow \mathcal{U}$ is a diffeomorphism, that $\pi\left(\mathcal{U}_{a} \cap Q\right)=P$, and that $\pi\left(\mathcal{U}_{a} \cap G^{s}\right)=\mathcal{U}_{a} \cap G^{s}=\left\{u \in G: u=u^{*}=u^{-1}\right.$ and $\left.a u=u a\right\}$.
\end{abstract}

Key words. Polar decomposition, $\mathrm{C}^{*}$-algebras, positive operators, projections.

AMS subject classifications. Primary 47A30, 47B15

1. Introduction. If $\mathcal{A}$ is the algebra of bounded linear operators in a Hilbert space $\mathcal{H}$, denote by $G$ the group of invertible elements of $\mathcal{A}$. Every $T \in G$ admits two polar decompositions

$$
T=U_{1} P_{1}=P_{2} U_{2}
$$

where $U_{1}, U_{2}$ are unitary operators (i.e. $U_{i}^{*}=U_{i}^{-1}$ ) and $P_{1}, P_{2}$ are positive operators (i.e $\left\langle P_{i} \xi, \xi\right\rangle>0$ for every $\xi \in \mathcal{H}$ ). It turns out that $U_{1}=U_{2}, P_{1}=\left(T^{*} T\right)^{1 / 2}$ and $P_{2}=\left(T T^{*}\right)^{1 / 2}$. We shall call $U=U_{1}=U_{2}$ the unitary part of $T$. Consider the map

$$
\pi: G \rightarrow \mathcal{U} \quad \pi(T)=U
$$

where $\mathcal{U}$ is the unitary group of $\mathcal{A}$. If $G^{+}$denotes the set of all positive invertible elements of $\mathcal{A}$, then every $A \in G^{+}$defines an inner product $\langle,\rangle_{A}$ on $\mathcal{H}$ which is equivalent to the original $\langle$,$\rangle ; namely$

$$
\langle\xi, \eta\rangle_{A}=\langle A \xi, \eta\rangle \quad(\xi, \eta \in \mathcal{H}) .
$$

* Received by the editors on 28 September 1999. Accepted for publication on 16 January 2000. Handling editor: Chandler Davis.

${ }^{\dagger}$ Instituto Argentino de Matemática, Saavedra 15, Piso 3, 1083 Buenos Aires, Argentina (gcorach@mate.dm.uba.ar). Partially supported by Fundación Antorchas, CONICET (PIP 4463/96), Universidad de Buenos Aires (UBACYT TX92 and TW49) and ANPCYT (PICT97-2259)

$\ddagger$ Instituto de Ciencias, UNGS, Roca 851, San Miguel, Buenos Aires, Argentina (amaestri@ungs.edu.ar)

$\S$ Departamento de Matemática, UNLP, calles 1 y 50, 1900 La Plata, Argentina (demetrio@mate.dm.uba.ar). Partially supported by CONICET (PIP 4463/96), Universidad de Buenos Aires (UBACYT TW49) and ANPCYT (PICT97-2259) 
Every $X \in \mathcal{A}$ admits an $A$-adjoint operator $X^{*_{A}}$, which is the unique $Y \in \mathcal{A}$ such that

$$
\langle X \xi, \eta\rangle_{A}=\langle\xi, Y \eta\rangle_{A} \quad(\xi, \eta \in \mathcal{H}) .
$$

It is easy to see that $X^{*_{A}}=A^{-1} X^{*} A$. Together with the definition of $*_{A}$ one gets the sets of A-Hermitian operators

$$
\mathcal{A}_{A}^{h}=\left\{X \in \mathcal{A}: X^{*_{A}}=X\right\}=\left\{X \in \mathcal{A}: A X=X^{*} A\right\},
$$

A-unitary operators

$$
\mathcal{U}_{A}=\left\{X \in G: X^{*_{A}}=X^{-1}\right\}=\left\{X \in \mathcal{A}: A X^{-1}=X^{*} A\right\}
$$

and A-positive operators

$$
G_{A}^{+}=\left\{X \in \mathcal{A}_{A}^{h} \cap G:\langle X \xi, \xi\rangle_{A} \geq 0 \quad \forall \xi \in \mathcal{H}\right\}
$$

As in the "classical" case, i.e. $A=I$, we get two polar decompositions for each $T \in G$

$$
T=V_{1} R_{1}=R_{2} V_{2}
$$

with $V_{i} \in \mathcal{U}_{A}, R_{i} \in G_{A}^{+}, i=1,2$ and as before $V_{1}=V_{2}=V$. Thus, we get a map

$$
\pi_{A}: G \rightarrow \mathcal{U}_{A} \quad, \quad \pi_{A}(T)=V, \quad T \in G .
$$

This paper is devoted to a simultaneous study of the maps $\pi_{A}\left(A \in G^{+}\right)$, the way that

$$
\mathcal{U}_{A}, \quad G_{A}^{h}, \quad G_{A}^{+}
$$

intersect

$$
\mathcal{U}_{B}, \quad G_{B}^{h}, \quad G_{B}^{+}
$$

for different $A, B \in G^{+}$and the intersections of these sets with

$$
Q=\left\{S \in \mathcal{A}: S^{2}=I\right\} \quad \text { and } \quad P_{A}=\left\{S \in Q: S^{*_{A}}=S\right\}
$$

(reflections and $A$-Hermitian reflections of $\mathcal{A}$ ). The main result is the fact that, for every $A, B \in G^{+}$,

$$
\pi_{A} \mid \mathcal{U}_{B}: \mathcal{U}_{B} \rightarrow \mathcal{U}_{A}
$$

is a bijection. The proof of this theorem is based on the form of the positive solutions of the operator equation $X A X=B$ for $A, B \in G^{+}$. This identity was first studied by G. K. Pedersen and M. Takesaki [10] in their study of the Radon-Nykodym theorems in von Neumann algebras. As a corollary we get a short proof of the equality

$$
\pi_{A}\left(Q \cap \mathcal{U}_{B}\right)=P_{A}
$$

for every $A, B \in G^{+}$, which was proven in [1] as a $C^{*}$-algebraic version of results of Pasternak-Winiarski [8] on the analyticity of the map $A \mapsto P_{A}^{\mathcal{M}}$, where $P_{A}^{\mathcal{M}}$ is the $A$ orthogonal projection on the closed subspace $\mathcal{M}$ of $\mathcal{H}$. We include a parametrization of all solutions of Pedersen-Takesaki equation. The results are presented in the context of unital $\mathrm{C}^{*}$-algebras. 
2. Preliminaries. Let $\mathcal{A}$ be a unital $\mathrm{C}^{*}$-algebra, $G=G(\mathcal{A})$ the group of invertible elements of $\mathcal{A}, \mathcal{U}=\mathcal{U}(\mathcal{A})$ the unitary group of $\mathcal{A}, G^{+}=G^{+}(\mathcal{A})$ the set of positive invertible elements of $\mathcal{A}$ and $G^{s}=G^{s}(\mathcal{A})$ the set of positive selfadjoint elements of $\mathcal{A}$. Let $Q=Q(\mathcal{A})=\left\{\varepsilon \in G: \varepsilon^{2}=1\right\}$ be the space of reflections and

$$
P=P(\mathcal{A})=Q \cap G^{s}=Q \cap \mathcal{U}=\left\{\rho \in G: \rho=\rho^{*}=\rho^{-1}\right\}
$$

the space of orthogonal reflections, also called the Grassmann manifold of $\mathcal{A}$.

Each $g \in G$ admits two polar decompositions

$$
g=\lambda u=u^{\prime} \lambda^{\prime}, \quad \lambda, \lambda^{\prime} \in G^{+}, \quad u, u^{\prime} \in \mathcal{U} .
$$

In fact, $\lambda=\left(g g^{*}\right)^{1 / 2}, u=\left(g g^{*}\right)^{-1 / 2} g, \lambda^{\prime}=\left(g^{*} g\right)^{1 / 2}$ and $u^{\prime}=g\left(g^{*} g\right)^{-1 / 2}$. A simple exercise of functional calculus shows that $u=u^{\prime}$. We shall say that $u$ is the unitary part of $g$. Observe that in the decomposition $g=\lambda u$ (resp. $g=u \lambda^{\prime}$ ) the components $\lambda, u$ (resp. $u, \lambda^{\prime}$ ) are uniquely determined, for instance, if $\lambda u=\lambda_{o} u_{0}$, then $\lambda_{0}^{-1} \lambda=$ $u_{0} u^{-1}$ is a unitary element with positive spectrum: $\sigma\left(\lambda_{0}^{-1} \lambda\right)=\sigma\left(\lambda_{0}^{-1 / 2} \lambda \lambda_{0}^{1 / 2}\right)=$ $\sigma(\lambda) \subseteq \mathbb{R}^{+}$. Then $\lambda_{0}^{-1} \lambda=u_{0} u^{-1}=1$. The map

$$
\pi: G \rightarrow \mathcal{U} \quad \text { given by } \quad \pi(g)=u \quad(g \in G)
$$

is a fibration with very rich geometric properties (see [11], [2] and the references therein). We are interested in the way that the $0 \pi^{-1}(u)=G^{+} u=u G^{+}$intersect the base space of a similar fibration induced by a different involution. More precisely, each $a \in G^{+}$induces a $C^{*}$ involution on $\mathcal{A}$, namely

$$
x^{\# a}=a^{-1} x^{*} a .
$$

If $\mathcal{A}$ is represented in the Hilbert space $\mathcal{H}$, then $a \in G^{+}$induces the inner product $\langle,\rangle_{a}$ given by

$$
\langle\xi, \eta\rangle_{a}=\langle a \xi, \eta\rangle, \quad \xi, \eta \in \mathcal{H} .
$$

It is clear that $\langle x \xi, \eta\rangle_{a}=\left\langle\xi, x_{a}^{\#} \eta\right\rangle$ for all $x \in \mathcal{A}$ and $\xi, \eta$ in $\mathcal{H} . \mathcal{A}$ is a $\mathrm{C}^{*}$-algebra with this involution and with the norm $\|\cdot\|_{a}$ associated to $\langle,\rangle_{a},\|x\|_{a}=\left\|a^{1 / 2} x a^{-1 / 2}\right\|, x \in$ $\mathcal{A}$. For each $a \in G^{+}$, consider the unitary group $\mathcal{U}_{a}$ corresponding to the involution $\#$ :

$$
\mathcal{U}_{a}=\left\{g \in G: g^{\# a}=g^{-1}\right\}=\left\{g \in G: a^{-1} g^{*} a=g^{-1}\right\} .
$$

We shall study the restriction $\left.\pi\right|_{\mathcal{U}_{a}}$ and the way that different $\mathcal{U}_{a}, \mathcal{U}_{b}$ are set in $G$. Moreover, we shall also consider the a-hermitian part of $G$,

$$
G_{a}^{s}=\left\{g \in G: g^{\# a}=g\right\}=\left\{g \in G: a^{-1} g^{*} a=g\right\},
$$

the a-positive part of $G_{a}^{s}$

$$
G_{a}^{+}=\left\{g \in G_{a}^{s}: \sigma(g) \subseteq \mathbb{R}^{+}\right\}
$$


and the intersections of these sets when $a$ varies in $G^{+}$. The reader is referred to [7] and [5] for a discussion of operators which are hermitian for some inner product.

Observe that each $a \in G^{+}$induces a fibration $\pi_{a}: G \rightarrow \mathcal{U}_{a}$ with fibers homeomorphic to $G_{a}^{+}$. This paper can be seen in some sense as a simultaneous study of the fibrations $\pi_{a}, a \in G^{+}$.

Let us mention that, from an intrinsic viewpoint, $\mathcal{U}_{a}$ can be identified with $\mathcal{U}$. Indeed, consider the map $\varphi_{a}: \mathcal{A} \rightarrow \mathcal{A}$ given by

$$
\varphi_{a}(b)=a^{-1 / 2} b a^{1 / 2} \quad(b \in \mathcal{A}) .
$$

Then $\varphi_{a}(\mathcal{U})=\mathcal{U}_{a}, \varphi_{a}\left(G^{s}\right)=G_{a}^{s}$ and $\varphi_{a}\left(G^{+}\right)=G_{a}^{+}$, since $\varphi_{a}:(\mathcal{A}, *) \rightarrow\left(\mathcal{A}, \#_{a}\right)$ is an isomorphism of $\mathrm{C}^{*}$-algebras. We are concerned with the way in which the base space and fibers of different fibrations behave with respect to each other.

3. The polar decomposition. In [10], Pedersen and Takesaki proved a technical result which was relevant for their generalization of the Sakai's Radon-Nikodym theorem for von Neumann algebras [11]. More precisely, they determined the uniqueness and existence of positive solutions of the equation $T H T=K$ for $H, K$ positive bounded operators in a Hilbert space. We need a weak version of their result, namely when $H, K$ are positive invertible operators. In this case it is possible to give an explicit solution.

Lemma 3.1 ([10]). If $H, K$ are positive invertible bounded operators in a Hilbert space, the equation

$$
T H T=K
$$

has a unique solution, namely $T=H^{-1 / 2}\left(H^{1 / 2} K H^{1 / 2}\right)^{1 / 2} H^{-1 / 2}$.

Proof. Multiply (3) at left and right by $H^{1 / 2}$ and factorize

$$
H^{1 / 2} T H T H^{1 / 2}=\left(H^{1 / 2} T H^{1 / 2}\right)^{2} .
$$

Then we get the equation $\left(H^{1 / 2} T H^{1 / 2}\right)^{2}=H^{1 / 2} K H^{1 / 2}$. Taking (positive) square roots and using the invertibility of $H^{1 / 2}$ we get the result.

Returning to the map $\pi: G \rightarrow \mathcal{U}$, consider the fiber $\pi^{-1}(u)=\left\{\lambda u: \lambda \in G^{+}\right\}$. In order to compare the fibration $\pi$ with $\pi_{a}$, the following is the key result

Theorem 3.2. Let $a \in G^{+}$. Then, for every $u \in \mathcal{U}$ the fiber $\pi^{-1}(u)$ intersects $\mathcal{U}_{a}$ at a single point, namely $a^{-1 / 2}\left(a^{1 / 2} u a u^{-1} a^{1 / 2}\right)^{1 / 2} a^{-1 / 2} \cdot u$. In other words, the restriction $\left.\pi\right|_{\mathcal{U}_{a}}: \mathcal{U}_{a} \rightarrow \mathcal{U}$ is a homeomorphism.

Proof. If $g=\lambda u \in \mathcal{U}_{a}$ then $a^{-1} g^{*} a=g^{-1}$ is equivalent to $a^{-1} u^{-1} \lambda a=u^{-1} \lambda^{-1}$, so, after a few manipulations,

$$
\lambda a \lambda=u a u^{-1} .
$$

By Pedersen and Takesaki's result, there is a unique $\lambda \in G^{+}$which satisfies equation (4) for fixed $a \in G^{+}, u \in \mathcal{U}$, namely $\lambda=a^{-1 / 2}\left(a^{1 / 2} u a u^{-1} a^{1 / 2}\right)^{1 / 2} a^{-1 / 2}$. Thus, $\left(\pi \mid \mathcal{U}_{a}\right)^{-1}: \mathcal{U} \rightarrow \mathcal{U}_{a}$ is given by

$$
\left(\pi \mid \mathcal{U}_{a}\right)^{-1}(u)=a^{-1 / 2}\left(a^{1 / 2} u a u^{-1} a^{1 / 2}\right)^{1 / 2} a^{-1 / 2} \cdot u
$$


which obviously is a continuous map.

Let $a \in G^{+}$and consider the involution $\#_{a}$ defined in equation (1). It is natural to look at those reflections $\varepsilon \in Q$ which are $\#_{a^{-}}$orthogonal, i.e the so called $\#_{a^{-}}$ Grassmann manifold of $\mathcal{A}$. Let us denote this space by

$$
P_{a}=\left\{\varepsilon \in Q: \varepsilon=\varepsilon^{\# a}=\varepsilon^{-1}\right\}=Q \cap \mathcal{U}_{a}=Q \cap G_{a}^{s} .
$$

In [8], Pasternak-Winiarski studied the behavior of the orthogonal projection onto a closed subspace of a Hilbert space when the inner product varies continuously. Note that we can identify naturally the space of idempotents $q$ with the reflections of $Q$ via the affine map $q \mapsto \varepsilon=2 q-1$, which also maps the space of orthogonal projections onto $P$. Based on [8], a geometric study of the space $Q$ is made in [1], where the characterization $\pi\left(P_{a}\right)=P$ is given (proposition 5.1 of [1]). In the following proposition we shall give a new proof of this fact by showing that the homeomorphism $\left.\pi\right|_{\mathcal{U}_{a}}: \mathcal{U}_{a} \rightarrow \mathcal{U}$ maps $P_{a} \subseteq \mathcal{U}_{a}$ onto $P \subseteq \mathcal{U}$. Therefore the formula given in equation (5) for the inverse of $\left.\pi\right|_{\mathcal{U}_{a}}$ extends the formula given in proposition 5.1 of [1] for $\left(\left.\pi\right|_{P_{a}}\right)^{-1}$, since they must coincide on $P$.

Proposition 3.3. Let $a \in G^{+}$. Then $\pi\left(P_{a}\right)=\pi\left(Q \cap \mathcal{U}_{a}\right)=P$. Therefore $\left.\pi\right|_{P_{a}}: P_{a} \rightarrow P$ is a homeomorphism.

Proof. By the previous remarks, we just need to show that $\pi\left(P_{a}\right)=P$. Observe that if $\varepsilon \in Q$ then $\rho=\pi(\varepsilon) \in P$ : in fact, if $\varepsilon=\lambda \rho$ then $\varepsilon=\varepsilon^{-1}=\rho^{-1} \lambda^{-1}$; but, since the unitary part of $\varepsilon$ corresponding to both right and left polar decompositions coincide, we get $\rho^{-1}=\rho$. Then $\rho^{*}=\rho^{-1}=\rho$ and $\rho \in P$. Thus, $\pi\left(\mathcal{U}_{a} \cap Q\right) \subseteq P$.

Let $\alpha=\left(\pi \mid \mathcal{U}_{a}\right)^{-1}: \mathcal{U} \rightarrow \mathcal{U}_{a}$. Then by $(5)$

$$
\alpha(u)=a^{-1 / 2}\left(a^{1 / 2} u a u^{-1} a^{1 / 2}\right)^{1 / 2} a^{-1 / 2} \cdot u .
$$

In order to prove the result we need to show that if $\rho \in P$ then $\alpha(\rho) \in P_{a}=Q \cap \mathcal{U}_{a}$, i.e. $\alpha(\rho) \in Q$. Indeed,

$$
\begin{aligned}
\alpha(\rho)^{2} & =a^{-1 / 2}\left(a^{1 / 2} \rho a \rho a^{1 / 2}\right)^{1 / 2} a^{-1 / 2} \rho a^{-1 / 2}\left(a^{1 / 2} \rho a \rho a^{1 / 2}\right)^{1 / 2} a^{-1 / 2} \rho \\
& =a^{-1 / 2}\left(\left(a^{1 / 2} \rho a^{1 / 2}\right)^{2}\right)^{1 / 2}\left(a^{1 / 2} \rho a^{1 / 2}\right)^{-1}\left(\left(a^{1 / 2} \rho a^{1 / 2}\right)^{2}\right)^{1 / 2} a^{-1 / 2} \rho .
\end{aligned}
$$

Thus, applying the continuous functional calculus (see e.g. [9]) to the selfadjoint element $a^{1 / 2} \rho a^{1 / 2}$, if $f(t)=|t|=\left(t^{2}\right)^{1 / 2}$ and $g(t)=\frac{1}{t}, t \in \mathbb{R} \backslash\{0\}$,

$$
\begin{aligned}
(\lambda \rho)^{2} & =a^{-1 / 2} f\left(a^{1 / 2} \rho a^{1 / 2}\right) g\left(a^{1 / 2} \rho a^{1 / 2}\right) f\left(a^{1 / 2} \rho a^{1 / 2}\right) a^{-1 / 2} \rho \\
& =a^{-1 / 2}\left[f\left(a^{1 / 2} \rho a^{1 / 2}\right)\right]^{2} g\left(a^{1 / 2} \rho a^{1 / 2}\right) a^{-1 / 2} \rho \\
& =a^{-1 / 2}\left(a^{1 / 2} \rho a^{1 / 2}\right) a^{-1 / 2} \rho=1 .
\end{aligned}
$$

3.1. Positive parts. In order to complete the results on the relationship between polar decomposition and inner products, consider the complementary map of the decomposition $g=\lambda u$, namely

$$
\pi^{+}: G \rightarrow G^{+}, \quad \pi^{+}(g)=\left(g g^{*}\right)^{1 / 2}, \quad(g \in G) .
$$


Of course, there is another "complementary map", namely $g \mapsto\left(g^{*} g\right)^{1 / 2}$ corresponding to the decomposition $g=u \lambda^{\prime}$. We shall see that for every $a \in G^{+}$, the restriction

$$
\left.\pi^{+}\right|_{G_{a}^{+}}: G_{a}^{+} \rightarrow G^{+}
$$

is a homeomorphism. Indeed, given $\mu \in G^{+}$, consider the polar decomposition $a \mu=$ $\lambda u$, with $\lambda \in G^{+}$and $u \in \mathcal{U}$. Then $a^{-1} \lambda=\mu u^{*}$, so $\pi^{+}\left(a^{-1} \lambda\right)=\mu$ and $a^{-1} \lambda \in G_{a}^{+}$, since $a^{-1}\left(a^{-1} \lambda\right)^{*} a=a^{-1} \lambda$ and the spectrum $\sigma\left(a^{-1} \lambda\right) \subseteq \mathbb{R}^{+}$. Note that

$$
\left(\left.\pi^{+}\right|_{G_{a}^{+}}\right)^{-1}(\mu)=a^{-1} \pi^{+}(a \mu),
$$

which is clearly a continuous map. An interesting rewriting of the above statement is the following:

Proposition 3.4. If $\mathcal{A}$ is a unital $C^{*}$-algebra and $a, \lambda \in G^{+}$, then there exists a unique $u \in \mathcal{U}$ such that $a \lambda u \in G^{+}$.

Proof. Indeed, if $g=\left(\left.\pi^{+}\right|_{G_{a}^{+}}\right)^{-1}(\lambda) \in G_{a}^{+}$and $u=\pi(g)$, then $\lambda u=g \in G_{a}^{+}$means exactly that $a \lambda u \in G^{+}$.

It is worth mentioning that $x \in G$ is the unique positive solution of PedersenTakesaki equation $x a x=b$ if and only if $a^{1 / 2} x b^{-1 / 2} \in \mathcal{U}$. Changing $a, b$ by $a^{2}, b^{-2}$ respectively, we can write Lemma 3.1 as follows:

Proposition 3.5. If $\mathcal{A}$ is a unital $C^{*}$-algebra and $a, b \in G^{+}$, then there exists a unique $x \in G^{+}$such that $a x b \in \mathcal{U}$.

3.2. Products of positive operators. The map $\Theta: G^{+} \times G^{+} \rightarrow \mathcal{U}$ given by

$$
\Theta(a, b)=a x b=a\left(a^{-1}\left(a b^{2} a\right)^{1 / 2} a^{-1}\right) b=\left(a b^{2} a\right)^{1 / 2} a^{-1} b, \quad a, b \in G^{+},
$$

is not surjective: in fact, the image of $\Theta$ consists of those unitary elements which can be factorized as a product of three positive elements. On one side $\Theta(a, b)=a x b \in \mathcal{U}$ is the product of three elements of $G^{+}$. On the other side, if $a x b \in \mathcal{U}$ then by Pedersen-Takesaki's result $x$ is the unique positive solution of $x a^{2} x=b^{-2}$.

It is easy to show that $-1 \in \mathcal{U}$ can not be decomposed as a product of four positive elements. See [12] and [13] for a complete bibliography on these factorization problems. See [3] for more results on factorization of elements of $G$ and characterizations of $P_{n}=\left\{a_{1} \ldots a_{n}: a_{i} \in G^{+}\right\}$, at least in the finite dimensional case.

3.3. Parametrization of the solutions of Pedersen-Takesaki equations. Given $a, b \in G^{+}$, denote by $m=\left|b^{1 / 2} a^{1 / 2}\right|=\left(a^{1 / 2} b a^{1 / 2}\right)^{1 / 2}$. Then the set of all solutions of the equation $x a x=b$ is

$$
\left\{a^{-1 / 2} m \varepsilon a^{-1 / 2}: \varepsilon \in Q \quad \text { and } \quad \varepsilon m=m \varepsilon\right\} .
$$

In fact, $x a x=b$ if and only if $\left(a^{1 / 2} x a^{1 / 2}\right)^{2}=m^{2}$ and the set of all solutions of $x^{2}=c^{2}$ for $c \in G^{+}$is $\left\{c \varepsilon: \varepsilon^{2}=1\right.$ and $\left.\varepsilon c=c \varepsilon\right\}$. The singular case, which is much more interesting, deserves a particular study that we intend to do in a forthcoming paper. 
4. Intersections and unions. For any selfadjoint $c \in \mathcal{A}$ we shall consider the relative commutant subC $^{*}$-algebra

$$
\mathcal{A}_{c}=\mathcal{A} \cap\{c\}^{\prime}=\{d \in \mathcal{A}: d c=c d\}
$$

and denote by $\mathcal{U}\left(\mathcal{A}_{c}\right)=\mathcal{A}_{c} \cap \mathcal{U}$, the unitary group of $\mathcal{A}_{c}$ and, analogously $G^{s}\left(\mathcal{A}_{c}\right)$, $G^{+}\left(\mathcal{A}_{c}\right), Q\left(\mathcal{A}_{c}\right)$ and $P\left(\mathcal{A}_{c}\right)$.

The space $G^{s}$ has a deep relationship with $Q$ (in [4] there is a partial description of it). Here we only need to notice that the unitary part of any $c \in G^{s}$ also belongs to $P$. Indeed, if $\lambda \rho$ is the polar decomposition of $c$, then $\lambda \rho=c=c^{*}=\rho^{*} \lambda$. By the uniqueness of the unitary part, $\rho=\rho^{*}=\rho^{-1} \in P$. Observe also that $\rho \lambda=\lambda \rho$. Moreover, since $\lambda=|c|=\left(c^{2}\right)^{-1}$, then $\rho=f(c)$ where $f(t)=t|t|$. So $\rho c=c \rho$.

TheOREM 4.1. Let $\mathcal{A}$ a unital $C^{*}$-algebra and $a \in G^{+}$. Then

$$
\mathcal{U}_{a} \cap G^{s}=P\left(\mathcal{A}_{a}\right)=\{u \in P: a u=u a\} .
$$

Proof. By the previous remarks, if $b \in G^{s} \cap \mathcal{U}_{a}$ and $b=\lambda \rho$ is its polar decomposition, then $\rho \in P$ and $\rho \lambda^{-1}=a^{-1} \rho \lambda a$. Using that $\rho \lambda=\lambda \rho$ we get easily

$$
\lambda^{-1} a \lambda^{-1}=\rho a \rho=\lambda a \lambda .
$$

By the uniqueness of the positive solution, $\lambda=\lambda^{-1}$ and, since $\lambda \in G^{+}$, this means that $\lambda=1$. Thus $a=\rho a \rho$ and then $\rho \in P\left(\mathcal{A}_{a}\right)$. Conversely, if $\rho \in P\left(\mathcal{A}_{a}\right)$, then $\rho \in \mathcal{U}_{a}$, since $a^{-1} \rho^{*} a=a^{-1} \rho a=\rho=\rho^{-1}$.

REMARK 4.2. Let $a \in G^{+}$. Then easy computations show that

1. $\mathcal{U}_{a} \cap \mathcal{U}=\mathcal{U} \cap \mathcal{A}_{a}=\mathcal{U}\left(\mathcal{A}_{a}\right)$.

2. $G_{a}^{s} \cap G^{s}=G^{s} \cap \mathcal{A}_{a}=G^{s}\left(\mathcal{A}_{a}\right)$.

3. $G_{a}^{+} \cap G^{+}=G^{+} \cap \mathcal{A}_{a}=G^{+}\left(\mathcal{A}_{a}\right)$.

4. $\mathcal{U}_{a} \cap G^{+}=\{1\}$.

We shall give two proofs of item 4 :

First proof: $\pi\left(G^{+}\right)=\{1\}$ but $\pi$ restricted to $\mathcal{U}_{a}$ is one to one.

Second proof: if $x \in \mathcal{U}_{a} \cap G^{+}$, then its spectrum $\sigma(x) \subseteq S^{1} \cap \mathbb{R}^{+}=\{1\}$; on the other side, $x$ is normal with respect to the involutions $\#_{a}$, so $x$ is a normal element such that $\sigma(x)=\{1\}$ and it must be $x=1$.

Let $b \in G^{+}$. Recall that the map $\varphi_{b}$ defined in (2) changes the usual involution by $\#_{b}$ and also all the corresponding spaces $\left(\operatorname{e.g} \varphi_{b}\left(G^{s}\right)=G_{b}^{s}\right)$.

Lemma 4.3. Let $b \in G^{+}$. Then for any $c \in G^{+}$, if $d=b^{-1 / 2} c b^{-1 / 2}$,

$$
\varphi_{b}\left(\mathcal{U}_{d}\right)=\mathcal{U}_{c}, \quad \varphi_{b}\left(G_{d}^{s}\right)=G_{c}^{s}, \quad \text { and } \quad \varphi_{b}\left(G_{d}^{+}\right)=G_{c}^{+}
$$

Proof. Notice that $\mathcal{U}_{d}=\varphi_{d}(\mathcal{U})$, so $\varphi_{b}\left(\mathcal{U}_{d}\right)=\varphi_{b} \circ \varphi_{d}(\mathcal{U})$. But $\varphi_{b} \circ \varphi_{d}=\varphi_{c} \circ \operatorname{Ad}\left(u^{*}\right)$ where $u=\pi\left(d^{1 / 2} b^{1 / 2}\right)$, since $c=\left|d^{1 / 2} b^{1 / 2}\right|^{2}$ and $d^{1 / 2} b^{1 / 2}=u c^{1 / 2}$. As $A d_{u^{*}}(\mathcal{U})=\mathcal{U}$ ( and the same happens for $G^{s}$ and $G^{+}$), we get $\varphi_{b}\left(\mathcal{U}_{d}\right)=\mathcal{U}_{c}$ and the other two identities.

Then we can generalize the results above for any pair $a, b \in G^{+}$instead of $a$ and 1:

Corollary 4.4. Let $a, b \in G^{+}$and $c=b^{-1 / 2} a b^{-1 / 2}$. Then 
1. $\mathcal{U}_{a} \cap G_{b}^{s}=\varphi_{b}\left(\mathcal{U}_{c} \cap G^{s}\right)=b^{-1 / 2}\left(P\left(\mathcal{A}_{c}\right)\right) b^{1 / 2}$.

2. $\mathcal{U}_{a} \cap \mathcal{U}_{b}=\varphi_{b}\left(\mathcal{U}_{c} \cap \mathcal{U}\right)=b^{-1 / 2} \mathcal{U}\left(\mathcal{A}_{c}\right) b^{1 / 2}$.

3. $G_{a}^{s} \cap G_{b}^{s}=\varphi_{b}\left(G_{c}^{s} \cap G^{s}\right)=b^{-1 / 2} G^{s}\left(\mathcal{A}_{c}\right) b^{1 / 2}$.

4. $G_{a}^{+} \cap G_{b}^{+}=\varphi_{b}\left(G_{c}^{+} \cap G^{+}\right)=b^{-1 / 2} G^{+}\left(\mathcal{A}_{a}\right) b^{1 / 2}$.

5. $\mathcal{U}_{a} \cap G_{b}^{+}=\varphi_{b}\left(\mathcal{U}_{c} \cap G^{+}\right)=\{1\}$.

Proof. Use Proposition 4.1, Remark 4.2 and Lemma 4.3.

In the following proposition we describe the set of elements of $\mathcal{A}$ which are unitary (resp. positive, Hermitian) for some involution $\#_{a}\left(a \in G^{+}\right)$. We state the result without proof.

Proposition 4.5. If $\mathcal{A}$ is a unital $C^{*}$-algebra, the following identities hold:

$$
\begin{gathered}
\bigcup_{a \in G^{+}} \mathcal{U}_{a}=\bigcup_{g \in G} g \mathcal{U} g^{-1}=\bigcup_{a \in G^{+}} a \mathcal{U} a^{-1}, \\
\bigcup_{a \in G^{+}} G_{a}^{+}=\bigcup_{g \in G} g G^{+} g^{-1}=\bigcup_{a \in G^{+}} a G^{+} a^{-1}=G^{+} G^{+},
\end{gathered}
$$

where $G^{+} G^{+}=\left\{a b: a, b \in G^{+}\right\}$and

$$
\bigcup_{a \in G^{+}} G_{a}^{s}=\bigcup_{g \in G} g G^{s} g^{-1}=\bigcup_{a \in G^{+}} a G^{s} a^{-1}=G^{s} G^{+}=G^{+} G^{s} .
$$

The following example shows that there is no obvious spectral characterization of these subsets of $G$ : if $x$ is nilpotent, then $1+x$ does not belong to any of them but $\sigma(1+x)=\{1\} \subseteq \mathbb{R}, \mathbb{R}^{+}$and the circle $S^{1}$.

4.1. Final geometric remarks. All subsets of $\mathcal{A}$ studied in this paper have a rich structure as differential manifolds. The reader is referred to [6] and [2] for the case of $\mathcal{U}$ and to [4] (and the references therein) for $Q, P, G^{s}$ and $G^{+}$. The map $\varphi_{a}$ defined in equation (2) is clearly a diffeomorphism which allows to get all the information on $\mathcal{U}_{a}, G_{a}^{s}, G_{a}^{+}$from that available on $\mathcal{U}, G^{s}, G^{+}$, respectively. The main results of the paper say that the map $\pi$ is a diffeomorphism between $\mathcal{U}_{a}$ and $\mathcal{U}, P_{a}$ and $P$ and so on.

\section{REFERENCES}

[1] Andruchow E., Corach G. and Stojanoff D.; Geometry of oblique projections, Studia Mathematica 137:61-79, 1999.

[2] Atkin C. J., The Finsler geometry of groups of isometries of Hilbert spaces, Journal of the Australian Mathematical Society (Series A) 42:196-222, 1987.

[3] Ballantine C. S., Products of positive definite matrices IV, Linear Algebra and its Applications $3: 79-114,1970$

[4] Corach G.; Operator inequalities, geodesics and interpolation, in Zemanek, Jaroslav (ed.), Functional analysis and operator theory. Proceedings of the 39th semester at the Stefan Banach International Mathematical Center in Warsaw, Poland, held March 2-May 30, 1992. Warsaw: Polish Academy of Sciences, Banach Cent. Publ. Vol. 30, pp. 101-115, 1994.

[5] Dieudonné J.; Quasi-hermitian operators, in Proc. Internat. Symp. Linear Spaces, Jerusalem, pp. 115-122, 1961. 
[6] Harpe, P. de la; Classical Banach-Lie algebras and Banach-Lie groups in Hilbert space, Lecture Notes in Mathematics 285, Springer Verlag, Berlin-New York, 1972.

[7] Lax P. D.; Symmetrizable linear transformations, Communications in Pure and Applied Mathematics 7:633-647, 1954.

[8] Pasternak-Winiarski Z.; On the dependence of the orthogonal projector on deformations of the scalar product, Studia Mathematica 128:1-17, 1998.

[9] Pedersen G. K.; Analysis Now, Springer-Verlag, New York, 1989

[10] Pedersen G. K. and Takesaki M.; The operator equation THT $=K$, Proceedings of the American Mathematical Society 36:311-312, 1972.

[11] Pedersen G. and Takesaki M.; The Radon Nikodym theorem for von Neumann algebras, Acta Mathematica 130:53-87, 1973.

[12] Phillips, N. C.; Every invertible Hilbert space operator is a product of seven positive operators, Canadian Mathematical Bulletin 38:230-236, 1995.

[13] Wu, P. Y.; The operator factorization problem, Linear Algebra and its Applications 117:35-63, 1989. 